\title{
Preface
}

\section{Precision Medicine and PET/ Computed Tomography: Emerging Themes for Future Clinical Practice}

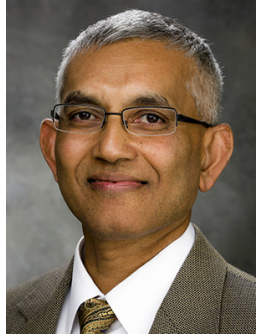

Rathan M. Subramaniam, MD, PhD, MPH Editor

Dr R.M. Subramaniam is supported by $\mathrm{U} 10$ CA180870.

In this second issue of a two-part series of PET Clinics, which is dedicated to collate the immense value of PET/CT for implementation of precision medicine, we cover esophageal cancer, gastric cancer, and pancreatic cancer, including neuroendocrine tumors, melanoma, sarcoma, uterine and ovarian cancers, cardiovascular disorders, and PET/MR imaging. Emerging themes include nuclear medicine theragnostics, PET/ MR imaging, and artificial intelligence and data analytics.

The neuroendocrine tumors paved the first wave of nuclear medicine theragnostics: diagnosis with ${ }^{68} \mathrm{Ga}$ DOTATATE PET/CT facilitating targets with expression of somatostatin receptors and peptide receptor radionucleide therapy with ${ }^{177}$ Lu DOTATATE, a beta emitter. ${ }^{68} \mathrm{Ga}$ DOTATATE was approved by the US Food and Drug Administration (FDA) in 2016 and is now reimbursed by the US Centers for Medicare and Medicaid Services. In a recently concluded randomized controlled phase 3 trial of ${ }^{177}$ Lu-DOTATATE for midgut neuroendocrine tumors, 229 patients, ${ }^{1}$ who had welldifferentiated, metastatic midgut neuroendocrine tumors, received either ${ }^{177}$ Lu-DOTATATE (116 patients) at a dose of $7.4 \mathrm{GBq}$ every 8 weeks (four intravenous infusions, plus best supportive care including octreotide long-acting repeatable [LAR] administered intramuscularly at a dose of $30 \mathrm{mg}$ ) ( ${ }^{177}$ Lu-DOTATATE group) or octreotide LAR alone (113 patients) administered intramuscularly at a dose of $60 \mathrm{mg}$ every 4 weeks (control group). The estimated rate of progression-free survival at month 20 was $65.2 \%$ in the ${ }^{177}$ Lu-DOTATATE group and $10.8 \%$ in the control group. The response rate was $18 \%$ in the ${ }^{177}$ Lu-DOTATATE group versus $3 \%$ in the control group $(P<.001)$. In the planned interim analysis of overall survival, 14 deaths occurred in the ${ }^{177}$ Lu-DOTATATE group and 26 in the control group $(P=.004)$. This is a landmark trial for the field of theragnostic nuclear medicine and molecular imaging and likely will lead to US FDA approval of ${ }^{177}$ Lu-DOTATATE therapy in the future.

PET/MR imaging provides superior soft tissue characterization than PET/CT can render. $\mathrm{PET} / \mathrm{MR}$ imaging with precision $\mathrm{PET}$ radiopharmaceuticals can provide multiparametric information about tumors for diagnosis and prognosis and in combination with theragnostic 
radiopharmaceuticals would make an impact on patient outcomes. More efforts in organizing prospective clinical studies to demonstrate the value of PET/MR imaging, improving work flow and implementations, attenuation correction improvements to reduce biases in quantitative measurements, and advanced quantitative analytics are necessary to reap the full benefits of this emerging clinical modality.

Impact of artificial intelligence using medical imaging, including PET/CT and PET/MR imaging, will facilitate precision medicine. Medical imaging data comprise about $90 \%$ of all medical data, which provide enormous opportunities exploring artificial intelligence in tumor detection, making precision therapy decisions and prognosis. Within the next 10 to 20 years, artificial intelligence will impact the medical imaging workflow and patient management.
The future of PET/CT, PET/MR imaging, and nuclear medicine theragnostics is as bright as ever we have seen.

Rathan M. Subramaniam, MD, PhD, MPH Department of Radiology The University of Texas Southwestern Medical Center 5323 Harry Hines Boulevard Dallas, TX 75390-8896, USA

E-mail address:

rathan.subramaniam@UTsouthwestern.edu

\section{REFERENCE}

1. Strosberg J, El-Haddad G, Wolin E, et al. Phase 3 trial of 177Lu-dotatate for midgut neuroendocrine tumors. N Engl J Med 2017;376(2):125-35. 